\title{
Levantamiento arquitectónico de la Puerta de Antioquía (Alepo)
}

\section{Architectural survey of the Antioch gate (Aleppo)}

\author{
M.A. Núñez ${ }^{(*)}$, F. Buill(*), J. Regot(**), A. de Mesa ${ }^{(* *)}$ \\ RESUMEN

\section{SUMMARY}

Desde el siglo XVII hasta la actualidad los levantamientos arquitectónicos han cambiado enormemente debido al avance en las técnicas e instrumentos de medida. En la última década las aplicaciones que permiten trabajar sin demasiadas dificultades con datos 3D han desencadenado la necesidad de generar y visualizar datos de este modo. Se ha pasado de técnicas de captura discreta (métodos topográficos, fotogrametría analógica y analítica) donde el operador debía decidir que elemento tomaba, a técnicas de captura masiva como la fotogrametría digital y el láser escáner terrestre, donde el instrumento captura automáticamente la información 3D. Este avance ha cambiado la forma de representar elementos arquitectónicos, evolucionando en paralelo al desarrollo de la técnica y pasando de representaciones $2 \mathrm{D}$ a $3 \mathrm{D}$, en las que se dispone de información de color y textura además.

Toda esta información puede tener dos claras aplicaciones: la obtención de modelos virtuales realistas de las ciudades, de claro valor urbanístico y constituir la información clave para actuación a nivel de edificio (catalogación, proyectos de rehabilitación,...).

283-2

Palabras clave: Fotogrametría; láser escáner; levantamiento arquitectónico; restauración; rehabilitación; modelos 3D.
Since the 17th Century to the present architectural survey has undergone an important change due to the continued progress in measuring techniques and instruments. In the last decade the increase of applications that let you work without too much difficulty with 3D data has triggered the need to generate and display data in this way. In a few years the capture techniques focused on measuring a few point very well defined (survey methods, analytical photogrammetry) have been superseded by the massive capture techniques, among them digital photogrammetry and terrestrial laser scanner. This breakthrough has changed the representation of architectural elements, which has evolved in parallel with the development of technology, from $2 D$ to $3 D$, in which we can also have color information and texture.

All this information can have two clear applications: the obtaining realistic virtual models of cities, and information to address any action on buildings (cataloging, rehabilitation projects,...).

Keywords: Photogrammetry; laser scanning; architectural survey; restoration; rehabilitation; $3 D$ models.

\footnotetext{
(*) ETCG-Universitat Politècnica de Catalunya, Barcelona (España).

(**) EGA I-Universitat Politècnica de Catalunya, Barcelona (España).

Persona de contacto/Corresponding author: m.amparo.nunez@upc.edu (M.A. Nuñez)
} 


\section{INTRODUCCIÓN}

El 16 de noviembre de 1972 la Conferencia General de la Organización de las Naciones Unidas, la Ciencia y la Cultura en su decimoséptimo período de sesiones en París, aprobó y promulgó la "Convención para la Protección del Patrimonio Mundial Cultural y Natural", cuyo objetivo es promover la identificación, protección y preservación del patrimonio cultural y natural de todo el mundo (1).

El patrimonio cultural engloba, entre otros elementos, todos los bienes materiales que se significan por su relación con la cultura de un pueblo.

La pérdida del patrimonio debida a desastres naturales como las inclemencias climáticas, los terremotos o las inundaciones y su destrucción producida por la mano del hombre a causa de intereses particulares o colectivos, son las causas que exigen la necesidad de disponer de los datos suficientes que permitan la reconstrucción de los bienes de interés que hayan sufrido deterioros totales o parciales.

También es patente la necesidad de analizar y detectar posibles malformaciones o degradaciones de los elementos constructivos de todos estos bienes debidos a los agentes contaminantes, el cambio climático y a la polución en las ciudades y de esta forma poder controlar la solidez de la edificación y aplicar las medidas oportunas que permitan su conservación.

Por estas razones es necesaria la catalogación y control de aquellos bienes que tienen un interés especial de esta manera se evitará, en caso de destrucción, la pérdida de estos bienes ya que se podrán reconstruir y evitar su desaparición.

La catalogación debe ser lo más exhaustiva posible y los procesos que se deben seguir para alcanzar este fin parten de la captación de los datos existentes de forma que se pueda disponer de una información completa de cada uno de estos bienes culturales. El control total del estado de la edificación, su estabilidad, la degradación de materiales etc... permitirá poder aplicar las técnicas adecuadas para su recuperación.

Por ello es necesario la aplicación de técnicas y metodologías de trabajo que permitan obtener datos métricos, aspectos formales y características de los materiales de modo que se puedan hacer los análisis pertinentes para proceder a su rehabilitación, reparación o recuperación en caso de deterioro o destrucción y de este modo mantener en buen estado de conservación los bienes patrimoniales.
Actualmente las técnicas topográficas, la fotogrametría, el láser escáner, y los procesos de control y postproducción de la imagen fotográfica permiten obtener estos datos en tiempos de trabajo relativamente cortos.

El problema se presenta posteriormente en el tratamiento de los datos espaciales, principalmente si en el análisis de los aspectos formales de una edificación aparecen elementos compuestos por superficies complejas como es el caso, entre otros, de elementos constructivos formalizados con superficies regladas o bóvedas que debido a su antigüedad no responden a geometrías clásicas de la arquitectura. Para tales casos presentamos el empleo de métodos estadísticos que facilitan la determinación de estas superficies y permiten ofrecer modelos métricos y formales ajustados a una realidad parametrizable.

Un ejemplo de aplicación de estas técnicas para la detección de la degradación y las malformaciones constructivas de un valor patrimonial es el estudio que se ha realizado sobre la Puerta de entrada en la antigua ciudad de Alepo conocida como "Bab Antakya" objeto de trabajo en el proyecto RehabiMed (Rehabilitation of the Traditional Mediterranean Architecture), por su especial relevancia en la zona.

\section{METODOLOGÍA DE TRABAJO. INTRODUCCIÓN}

Para elaborar un levantamiento arquitectónico se pueden utilizar distintas técnicas de captura de información. En la actualidad se utilizan las técnicas de captura masiva de datos espaciales como el láser escáner (LiDAR, Light Detection And Ranging) y la fotogrametría digital.

En ambos casos es necesario georreferenciar los datos obtenidos, por lo que se ha de disponer de un apoyo topográfico.

\section{Fotogrametría}

La fotogrametría es un procedimiento de medida general que permite conseguir información métrica a partir imágenes fotográficas. La información extraída por este método puede ser bidimensional o tridimensional.

Las ventajas de utilización de la fotogrametría se basan principalmente en la sustitución del trabajo de campo por trabajo de gabinete, la posibilidad de determinar líneas continuas del modelo de trabajo que darán mayor precisión y homogeneidad, o la de tomar todo tipo de datos de objetos, incluso en movimiento (2). 
Para poder efectuar un trabajo fotogramétrico es necesario desarrollar las siguientes fases:

- Adquisición de datos, fundamentalmente mediante cámaras fotográficas e instrumentos topográficos

- Obtención de medidas y formas para el control de posiciones de los objetos a partir de la localización de puntos en las imágenes

- Aplicación de las transformaciones precisas, a partir de construcciones geométricas-analíticas y software para la restitución fotográfica.

Los métodos más utilizados en los levantamientos fotogramétricos arquitectónicos y arqueológicos son la restitución estereofotogramétrica, para conseguir resultados tridimensionales, y la rectificación y la ortoproyección para la representación de datos bidimensionales (3).

\section{Restitución}

En el caso de la restitución es necesario conseguir las coordenadas espaciales de puntos de algún elemento de la imagen, a partir de medidas efectuadas en un mínimo de dos fotografías de dicho objeto, en dos posiciones distintas. Las fases de trabajo son:

- La reconstrucción del haz perspectivo fotográfico, es decir la obtención de la forma del haz para las dos fotografías (orientación interna).

- La determinación de la posición de cada haz en relación a un sistema coordenado y la relación entre este sistema y el de referencia utilizado (orientación externa).

- La identificación y la intersección de los rayos homólogos de las fotografías para encontrar su posición en el espacio (restitución).

Las ecuaciones más utilizadas provienen de la relación existente entre el punto imagen medido sobre la perspectiva fotográfica, el centro perspectivo y el punto terreno del que proviene, y se conocen como ecuaciones de colinealidad [1] (Karara, 1989).

$$
\begin{aligned}
& x=f \frac{a_{11}\left(X-X_{0}\right)+a_{12}\left(Y-Y_{0}\right)+a_{13}\left(Z-Z_{0}\right)}{a_{31}\left(X-X_{0}\right)+a_{32}\left(Y-Y_{0}\right)+a_{33}\left(Z-Z_{0}\right)} \\
& y=f \frac{a_{21}\left(X-X_{0}\right)+a_{22}\left(Y-Y_{0}\right)+a_{23}\left(Z-Z_{0}\right)}{a_{31}\left(X-X_{0}\right)+a_{32}\left(Y-Y_{0}\right)+a_{33}\left(Z-Z_{0}\right)}
\end{aligned}
$$

donde $x$ e $y$ son las coordenadas fotográficas (refinadas y referidas al punto principal) del punto considerado, $X, Y$ y $Z$ las coordenadas del punto en el modelo (terreno) en el sistema absoluto, $X_{0^{\prime}} Y_{0}$ y $Z_{0}$ son las coor- denadas del centro proyectivo en el mismo sistema absoluto, $f$ es la distancia principal (focal de la cámara) y $a_{i j}$ las componentes de la matriz de rotación que relaciona ambos sistemas de referencia. El sistema es no lineal, y generalmente se trabaja con sistemas sobredeterminados por lo que es necesario resolver mediante un ajuste por mínimos cuadrados (MMCC) a partir de unos valores aproximados de las incógnitas y mediante iteraciones sucesivas.

El proceso de toma tradicional de datos a partir de imágenes fotográficas (determinación directa), así como en el caso de una perspectiva dibujada, el número de puntos es finito y se hace necesario interpolar posiciones para tener una información continua. Sin embargo en estereofotogrametría se puede trabajar por líneas continuas con lo que se acelera el proceso de reconocimiento de las formas en el espacio. En la actualidad los sistemas fotogramétricos digitales permiten, de manera automática, conseguir gran cantidad de información espacial mediante algoritmos de correlación de imágenes que identifican los puntos homólogos de las fotografías de un mismo objeto (4), obteniendo una nube de puntos similar a la que se obtendría mediante láser escáner terrestre (TLS).

\section{Rectificación}

En el caso de que únicamente sea necesaria información bidimensional, se puede utilizar la rectificación fotográfica. Este proceso consigue transformar o enderezar una perspectiva fotográfica obteniendo una imagen semejante a la que se conseguiría en la fotografía exactamente perpendicular al plano de interés.

Para poder conseguir la restitución de un modelo plano utilizando un solo fotograma es necesario conocer o poder determinar la relación que existe entre el sistema fotográfico y el sistema terreno, para ello podemos utilizar métodos basados en la geometría proyectiva. La transformación puede también obtenerse a partir de la relación de proyectividad [2], formulación que se extrae de la mostrada anteriormente y que relaciona sólo las coordenadas bidimensionales de los dos planos, en cualquier sistema de medida coordenado referido a cada uno (plano imagen y plano modelo), las expresiones son:

[2]

$$
\begin{aligned}
& X=\frac{a x+b y+c}{u x+v y+1} \\
& Y=\frac{d x+e y+f}{u x+v y+1}
\end{aligned}
$$


donde $X$ e $Y$ son las coordenadas del alzado o planta en el sistema terrestre, $x$ e $y$ son las coordenadas fotográficas del punto imagen y los parámetros $a, b, c \ldots$ son los determinados del sistema lineal, y se calculan mediante ajuste mínimo cuadrático a partir de conocer la relación entre más de 4 puntos.

\section{Ortofotografía}

Podemos definir ortofotografía como el método fotogramétrico de obtención de cartografía 2D, corrigiendo las deformaciones producidas en la imagen fotográfica por el relieve y la inclinación de la fotografía, mediante la corrección (rectificación diferencial) de la fotografía original. El resultado es una nueva imagen fotográfica digital corregida.

Esta técnica permite aprovechar el contenido informativo de la fotografía digital y realizar las mismas medidas que en un plano topográfico. Las principales características son la riqueza informativa, la exactitud métrica y la rapidez de producción (en el caso digital). Las aplicaciones más comunes son en elementos con numerosos planos y en modelos con formas complejas (5).

Tanto la fotogrametría digital como las nuevas técnicas de captura masiva láser escáner permiten obtener gran cantidad de información cuantitativa y cualitativa en muy poco tiempo.

\section{Láser escáner}

Los sensores láser escáner aerotransportados (ALS) se han aplicado a la obtención de modelos digitales y cartografía desde la década de los 90 del pasado siglo (6) (7) (8). Posteriormente, con su transformación a sensores terrestres (TLS) se han ido empleando ampliamente en aplicaciones arquitectónicas y arqueológicas.

Un láser escáner es un sistema de medición que, al igual que la fotogrametría, no necesita contacto directo con el modelo a levantar. El sensor está compuesto por un elemento que genera un haz de luz láser, caracterizado por su baja dispersión, y un sistema de barrido, horizontal y vertical, de modo que efectuando una serie de perfiles paralelos se consigue cubrir toda la superficie a levantar, en forma de rejilla de paso impuesto por el operador.

En función del sistema de medida empleado se pueden clasificar los sensores en tres tipos:

- Tiempo de vuelo (ToF). Consiste en medir la distancia a partir del tiempo que tar- da un fotodiodo en emitir y detectar una luz láser. Permiten obtener la situación de puntos en el espacio con una precisión alrededor de $5 \mathrm{~mm}$ para distancias de $50 \mathrm{~m}$.

- Diferencia de fase. Mide la diferencia de fase entre la onda emitida por el sensor y la recibida después de su reflexión en el objeto a medir. A esta medida se le añade el número completo de longitudes de onda existentes entre el instrumento y la superficie. La distancia de trabajo así como el tiempo invertido en cada barrido es inferior al caso anterior.

- Triangulación óptica. Se basa en el cálculo de las coordenadas espaciales a partir de la intersección directa (intersección de rectas desde dos puntos situados en una base). En este caso la distancia de trabajo se reduce considerablemente, aumentando la precisión alcanzada.

Como resultado del barrido sobre una superficie se obtiene una nube de puntos disponiendo para cada uno de ellos de sus coordenadas tridimensionales. Con la posibilidad de completar esta información con el valor de reflectividad y el color, dependiendo este último de la disponibilidad de tener una cámara fotográfica adaptada al láser escáner en la toma durante el levantamiento (en el caso más general).

Los TLS tiene como principal ventaja su gran operatividad en campo, permitiendo capturar millones de puntos en pocos minutos, lo cual se convierte en un inconveniente a la hora del tratamiento de los datos espaciales al tener que manipular un gran volumen de información lo que ralentiza cualquier operación posterior.

Para que este tipo de sistemas sea completamente operativo es necesario que se avance en la automatización de los procesos de segmentación que permita independizar superficies sobre las nubes de puntos y la substitución éstas por primitivas gráficas.

En la actualidad los programas comerciales permiten realizar esta operación de forma semiautomática para superficies tales como cilindros, esferas, planos,..., pero no para otros elementos presentes en las construcciones arquitectónicas como pueden ser elipsoides, conos, hiperboloides, paraboloides,... Este tipo de superficies generalmente se han venido ajustando mediante NURBS (Non-Uniform Rational Basis Spline) con el inconveniente de tener que asumir la imprecisión que se produce al elaborar estas superficies basándose en la situación de los datos extraídos por el escáner y que acumulan a las imprecisiones propias, los errores posicionales debidos a 
la diferenciación de colores o materiales, los puntos de superficie de barrido muy anguladas, así como puntos no pertenecientes a ésta (por ejemplo en oquedades). En el caso de trabajar con gran cantidad de datos espaciales (láser escáner,...) los problemas producidos por una mala captura o equivocación en la toma ocasiona una incertidumbre en la definición de la superficie. En este trabajo se propone un procedimiento mediante ajuste de superficies cuadráticas con métodos estadísticos que nos permitan por un lado un filtrado y segmentación de las diferentes superficies que componen el elemento y por otro la obtención de los parámetros que definen la forma paramétrica de la superficie y su clasificación (9) (10).

Para conseguir esta parametrización de superficies es necesario efectuar la segmentación de la nube de puntos inicial en todos aquellos elementos que la componen para lo que existen diversos métodos atendiendo a los datos que se emplean:

- De manera manual

- A partir de imágenes

- En función de datos derivados (como la pendiente y la orientación)

- Empleando otras condiciones a cumplir por los puntos.

En muchas ocasiones los parches segmentados inicialmente presentan puntos no pertenecientes a la superficie de estudio, añadidos por una mala clasificación a otros cercanos. El método empleado en la detección de estos puntos dependerá de su número, así por ejemplo si son escasos bastarán procedimientos basados en test estadísticos centrados en las matrices criterio, pero si se dan en mayor cantidad lo mejor es acudir a métodos robustos (9) (10).

En este trabajo se ha utilizado un método de ajuste de superficies basado en técnicas robustas para determinar y eliminar puntos considerados como espurios (método RANSAC).

\section{Método de ajuste}

Primero se realiza una segmentación aproximada de las superficies que conforman el objeto, posteriormente se procede al ajuste de la superficie, eliminando en este proceso aquellos puntos que contienen errores groseros de medida o simplemente no pertenecen a la superficie.

El método RANSAC (RANdom SAmple Consensus), es un método robusto desarroIlado para el ajuste de modelos paramétricos a nubes de puntos que pueden contener un alto nivel de ruido (10). Su principal problema es la cantidad de tiempo y recur- sos necesarios en su ejecución. Para evitar esto Schnabel (11), propone efectuar una exploración aleatoria mediante combinatoria, limitando en la medida de lo posible el número de datos a utilizar, dejando los más "importantes" y comprobando la verosimilitud de dicha selección con el resto de puntos. Seguidamente se efectúa un ajuste mínimo cuadrático.

Tras el ajuste se vuelven a analizar los resultados apoyándonos en test estadísticos basados en las matrices de criterio, obtenidas en el cálculo, con el objetivo de eliminar aquellos puntos que presenten errores considerables (por no pertenencia a la superficie a determinar).

\section{CASO DE ESTUDIO}

La ciudad de Alepo es una de las ciudades más antiguas del mundo, habitada desde 1800 AC, fue declarada Patrimonio Cultural de la UNESCO en 1986 debido a sus particulares características y como medida de protección después de que fueran demolidos parte de los edificios históricos por el Plan General de remodelación de la ciudad.

Desde principio de los años 90, del siglo pasado, han sido rehabilitados y renovados muchos edificios, monumentos e infraestructuras de la ciudad. Esto requirió muchos esfuerzos conjuntos de la municipalidad y las autoridades estatales, y se benefició de ayudas internacionales. En 1992 el gobierno alemán y la República Árabe de Siria empezaron a trabajar juntos para mejorar la capacidad de administración urbana del Municipio de Alepo. La restauración de la ciudad antigua de Alepo fue el objetivo final del proyecto conjunto (12).

La "Puerta de Antioquia" está situada al oeste de la ciudad y forma parte de la muralla que la rodea, siendo ésta una de las entradas a la ciudad antigua.

El conjunto arquitectónico de la "Puerta de Antioquia", (Figura 1), se compone de tres partes bien diferenciadas: dos torreones y un núcleo central. El torreón de la izquier-
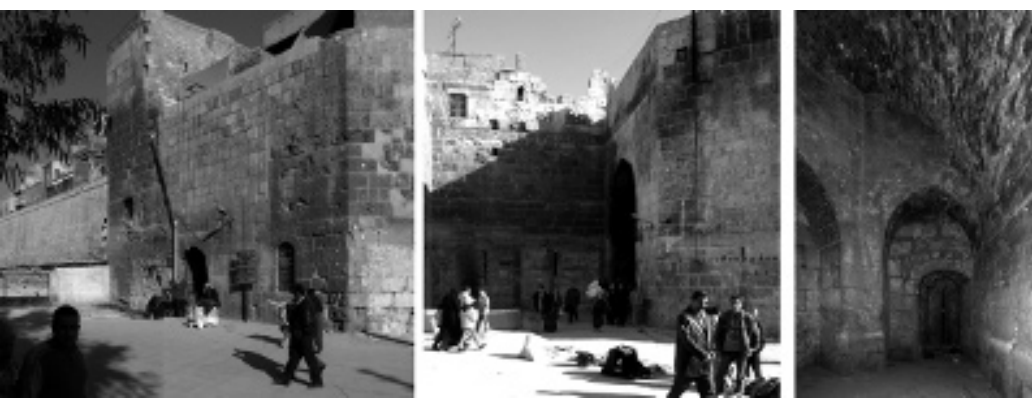
da, según se observa, con estancias añadidas en su parte superior encierra un espacio abovedado de tres naves con gran interés histórico tanto por su método constructivo como por su geometría formal. La torre de la derecha es la que da entrada a la ciudad antigua bajo un gran arco de piedra de carácter defensivo que emboca a un callejón en ángulo y que a su vez da acceso al zoco.

El conjunto queda limitado a su izquierda por el muro construido por los franceses en la época del Ilamado mandato francés (1920 40) que recubre parte de la antigua muralla.

En este contexto y en colaboración con el proyecto RehabiMed (Rehabilitation of the Traditional Mediterranean Architecture) y varios grupos de trabajo se realizó la toma de datos topográficos y fotográficos de la "Puerta de Antioquia" con un sistema láser escáner terrestre de la casa Riegl, apoyado con una estación total láser y diferentes cámaras fotográficas analógicas y digitales. El resultado de este trabajo ha sido el primer paso para el futuro proyecto de rehabilitación de la Puerta.

Así pues el reto consistía en obtener un modelo tridimensional lo más exacto posible del exterior e interior de las torres, así como la información gráfica de todos los paramentos verticales que componen la puerta, indicando las patologías que afectaban a los sillares: grietas, erosión, ...Para lo que ha sido necesario el empleo conjunto de diversas técnicas de medida.

En el caso de la obtención del modelo tridimensional la técnica empleada fue un levantamiento mediante láser escáner, en los siguientes epígrafes se explicará el proceso seguido.

En el caso de la información gráfica de los muros exteriores, se creyó que la mayor información la aportaría la elaboración de ortofotografías sobre las cuales se procedió al digitalizado, de este modo se dispone tanto de la información vectorial como de la imagen.

Para la georreferenciación y fusión de los datos espaciales obtenidos de las diferentes fuentes fue necesario establecer una serie de puntos de control, los cuales se dispusieron homogéneamente siguiendo un modelo similar a los puntos de Gruber para una toma fotogramétrica con apoyo continuo, de modo que siempre hubiera un número suficiente de puntos en cada toma. Las coordenadas de todos los puntos se obtuvieron tras el establecimiento de una red topográfica y observaciones efec- tuadas con una estación total sin prisma con una apreciación de $10^{c c}$ y precisión de $\pm(3 \mathrm{~mm}+\mathrm{ppm})$ en distancia.

La red topográfica estaba constituida por 14 bases distribuidas alrededor de la puerta y situadas a diferentes niveles. Tras la observación se realizó una compensación mediante ajuste por mínimos cuadrados para la obtención de las coordenadas finales de las bases y su error asociado, que en ningún caso supero $1 \mathrm{~cm}$ en planimetría y altimetría. Desde estas bases además de dar coordenadas a las dianas y puntos de control necesarios en el levantamiento fotogramétrico y mediante técnicas de láser escáner, se realizó el levantamiento de la mayor parte de las aristas que definen los diferentes planos de los paramentos. Para estos puntos se estima que el error absoluto asociado es de $1 \mathrm{~cm}$ tanto en altimetría como planimetría.

\section{Tomas fotográficas y trabajo fotogramétrico de gabinete}

Se realizaron dos tomas fotográficas, primero con una cámara semimétrica modelo Rollei 6006, de película tipo 120 (con un tamaño de $6 \times 6 \mathrm{~cm}$ ), con una placa reseau de 121 cruces esgrafiadas para control, con una distancia focal de $80,15 \mathrm{~mm}$, excepto en la parte central donde se encuentran las dos torres, debido a la corta distancia hacia el objeto, en este caso, la distancia focal utilizada fue de 40,49 mm. La película utilizada fue Kodak Ektachrome color en positivo con una sensibilidad de la emulsión de ISO 100. El solape entre las fotografías fue en todos los casos superior al $60 \%$.

La toma del entorno fotografiado se repitió con la cámara digital Nikon D200 (10,2 Mpx), con una óptica Zoom de 18200 mm VR DX, a la resolución máxima, calibrada para distintas focales. El trabajo fotogramétrico tuvo como objetivo obtener imágenes detalladas de la parte exterior de la muralla y de las dos torres que franquean la gran Puerta de Antioquía, que es la puerta de ingreso a la antigua ciudad de Alepo. Las escalas de captura estuvieron comprendidas entre $1 / 150$ y $1 / 250$.

La escala media de trabajo de las fotografías rectificadas y las ortofotografías fue de 1/50. Los alzados de la muralla se consiguieron utilizando estas fotografías mediante rectificaciones fotográficas con los puntos de control topográfico, seguidamente se mosaicaron corrigiendo la radiometría para obtener un producto de mayor calidad. Al mismo tiempo se efectuó una restitución de los pares fotográficos obteniendo un modelo espacial de los paños de la muralla que a su 
vez nos ha permitido la creación de un modelo de profundidades (MDE) que junto a las fotografías orientadas y mediante la ortoproyección de éstas la creación de una ortofotografía digital de resolución centimétrica.

Los problemas como zonas ocultas y definición de aristas se han resuelto con fotografías especiales y un proceso de orientación más cuidadoso al efectuar la orientación de las fotografías como si de un bloque fotogramétrico se tratara. En el caso de las diferencias radiométricas se han minimizado y corregido para dar una continuidad en los productos cartográficos finales.

Con la restitución fotogramétrica se ha obtenido un modelo tridimensional vectorial a partir del cual se han podido generar las posteriores representaciones gráficas, con una precisión algo inferior a la topográfica, alrededor de $1 \mathrm{~cm}$ en alzado y $2 \mathrm{~cm}$ en profundidad, (Figura 2).

En este caso los planos a representar se realizan a una escala de $1 / 50$ por lo que todos aquellos elementos del objeto que en la realidad tengan un tamaño superior a $1 \mathrm{~cm}$ deben ser restituidos ya que en los planos serán visibles.

En el desarrollo de este trabajo se han empleado dos software distintos, ambos con la capacidad de crear pares estereoscópicos a partir de los cuales se ha procedido a restituir el modelo tridimensional de cada una de las fachadas. El primer software empleado es Socet Set de BAE Systems, que conjuntamente con el módulo PRO600 permite conectar dicho programa con el software de CAD MicroStation. También se ha empleado el software de restitución Image Master de TOPCON el cual permite restituir directamente sobre los pares estereoscópicos y exportar estos en formato DXF.

\section{Trabajo de campo con el escáner láser}

Se requirieron unas 60 tomas con el láser escáner terrestre para poder recoger todos los datos accesibles de la edificación. El modelo de escáner láser utilizado fue el Riegl Z420i (13), este escáner tiene un campo visual con un amplio rango, i.e. desde $0^{\circ}$ a $360^{\circ}$ en horizontal, $-40^{\circ}$ a $+40^{\circ}$ en vertical, y un rango de distancia desde los $2 \mathrm{~m}$ hasta los $800 \mathrm{~m}$. La resolución de paso de malla se puede fijar a $0,0025^{\circ}$ en horizontal y en vertical. El método de medición utilizado por este instrumento es el tiempo de vuelo ToF (14) (15).

En cada estación del escáner para la zona exterior de la muralla se realizaron dos barridos: uno de baja resolución con un paso de resolución de $3,5 \mathrm{~cm}$ a $10 \mathrm{~m}$ de distancia y otro de alta resolución con una resolución de $1,5 \mathrm{~cm}$ a $10 \mathrm{~m}$.

Para obtener imágenes con información de color $R G B$ de cada barrido del escáner, se realizaron entre 10 y 12 capturas fotográficas con giro panorámico de $360^{\circ}$, mediante la cámara digital calibrada NIKON D100 (6 Mpx) acoplada sobre el cabezal del escáner.

La posibilidad de recoger los datos de reflectividad en longitud de onda infrarroja en las zonas más oscuras, permitió extraer datos que mediante las imágenes $R G B$ captadas por la fotografía digital no habían podido recoger y de este modo complementar los resultados del mosaico fotográfico, ya que con los datos de reflectividad se puede obtener la imagen del edificio en escala de grises que dependen de la intensidad de la señal recibida y complementa la información fotográfica obtenida de la zona. En la mayoría de los casos una gama de 256 niveles de grises son suficientes, en otros modelos es posible alcanzar los 512 ó 1028 niveles de intensidad, resultando muy fiable para detectar los cambios de materiales con mayor facilidad, y así definir las juntas de los sillares. Este hecho quedó claramente patente en el interior del pasillo que une ambas torres, donde la inexistencia de focos adecuados que permitieran una iluminación apropiada, provocó que en las imágenes fotográficas obtenidas fuera prácticamente imposible la distinción
2. Vista frontal del modelo vectorial resultante de la restitución.

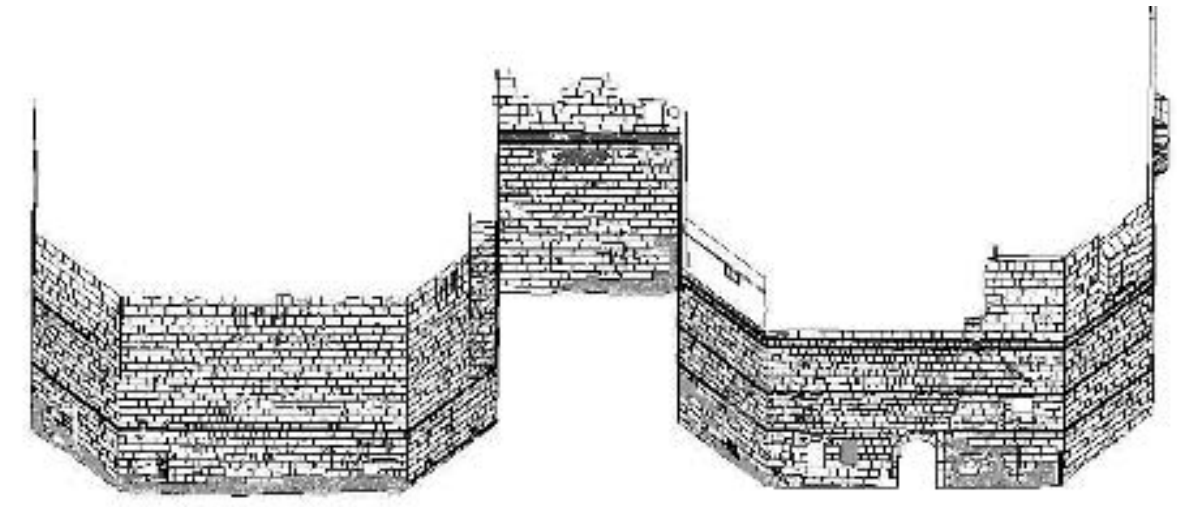


3. Izquierda nubes de puntos del interior de las torres, y a la derecha detalle de su valor de reflectividad de las juntas entre los sillares. Sin embargo visualizando la nube de puntos atendiendo a su valor de reflectividad estos límites quedaban claramente visibles por su diferente respuesta en la reflexión del rayo, (Figura 3).

A partir de los datos obtenidos con el láser escáner y para obtener la documentación y modelos necesarios se siguió el siguiente protocolo:

1. Asignación de color a las nubes de puntos. Para esta etapa del trabajo se ha utilizado el software RISCAN-Pro v1.2 $.1 b 17$ de Riegl.

2. Filtrado y limpieza de puntos ajenos al objeto. Se eliminan aquellos puntos que no forman parte de la edificación: vegetación, cables eléctricos, las personas, etc. Es muy complicado la eliminación de elementos que presentan un escaso "volumen" como pueden ser las telas de araña presentes en el interior de las torres.

3. La alineación de nubes. Se realizó en un doble proceso. Un primer encaje de las nubes parciales, (Figura 3), a partir de la identificación en éstas de dianas o elementos singulares dotados de coordenadas topográficas. Un registro automático posterior, a partir de métodos de mínimos cuadrados, permitió afinar la unión de las nubes captadas en el interior de la muralla (16) (17). Se estima que la precisión alcanzada en la nube de puntos final en absoluto es de $2,5 \mathrm{~cm}$ y relativo de $1,5 \mathrm{~cm}$.

4. El cálculo de las superficies que componen el modelo arquitectónico. El fin último es parametrizar las superficies que componen el objeto arquitectónico de forma que puedan ser sustituidas por primitivas gráficas. Usualmente se utilizan NURBS (Non Uniform Regular Beta Spline) para esta sustitución, produciéndose en este caso una indefinición geométrica al no poderse clasificar. Una solución es utilizar superficies cuádricas y efectuar su clasificación, si es posible, junto con los parámetros de los semiejes, origen y giros.
Además es recomendable conocer los parámetros que definen cada superficie para sustituir y reducir el peso de los modelos tridimensionales obtenidos por estos métodos de captura masiva de datos.

\section{La generación del modelo 3D teórico}

Para la generación de superficies se utilizó el software de Mc. Neel Rhinoceros v4.0 que permitió elaborar, a partir de los cálculos, las diferentes superficies que determinaban los paramentos verticales que soportaban las bóvedas así como las formas geométricas que conformaban las aspilleras interiores de la muralla. Las diferentes intersecciones entre superficies han definido las aristas de delimitación de cada superficie.

La comparación de estas superficies con las nubes de puntos permitió establecer las deformaciones debidas a asentamientos diferenciales de la estructura portante o a los defectos producidos en la ejecución de la construcción de los elementos. El software de Mc. Neel y de INUS Technology son herramientas que pueden determinar los resultados de aproximación a la forma real. La utilización de entidades de malla por triangulación, para formalizar una maqueta virtual, permite obtener un modelo muy aproximado a la realidad pero no un conocimiento de la geometría de las diferentes superficies que conforman los diferentes espacios. Para ello es necesario realizar un tratamiento más exhaustivo de las nubes de puntos, ajustando superficies correspondientes con primitivas gráficas como planos, cilindros,...

En el caso de estudio se ha utilizado el método RANSAC empleando dos tipos de ecuaciones en el ajuste, dependiendo de si el modelo era paramento de fachada o se trabajaba en las bóvedas. Para el primer caso empleamos la ecuación del plano [3] es:

[3]

$$
a_{11} x+a_{22} y+a_{33} z+a_{00}=0
$$
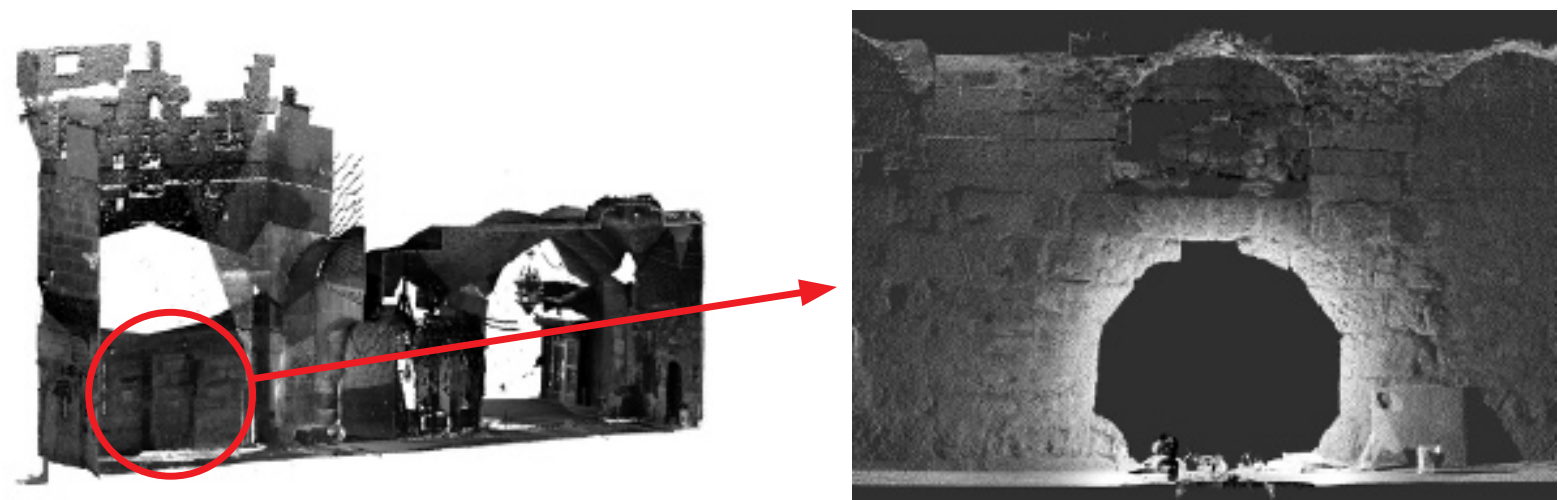
en el segundo caso la ecuación empleada es la general de las cuádricas [4] es:

[4]

$a_{11} x^{2}+a_{22} y^{2}+a_{33} z^{2}+2 a_{12} x y+2 a_{13} x z+$

$+2 a_{23} y z+2 a_{01} x+2 a_{02} y+2 a_{03} z+a_{00}=0$

Para el plano la tolerancia impuesta fue de $5 \mathrm{~cm}$, superior a la utilizada en las bóvedas que sólo llegaron a valores de $3 \mathrm{~cm}$. Esta diferencia se explica por el gran deterioro que han sufrido las paredes, con grandes oquedades y juntas abiertas, que no se presentan en las bóvedas.

Tras el ajuste se clasificaron las superficies cuádricas resultando cilindros, y se compararon éstos y los planos obtenidos con las nubes de puntos originales. Las discrepancias máximas se alcanzaban en las zonas donde existían oquedades, siendo la media de $3 \mathrm{~cm}$.

Los modelos obtenidos se completaron con secciones arquitectónicas generadas a partir de las nubes de puntos, (Figura 4), y que permitieron disponer de información de alta utilidad para afrontar el proyecto de rehabilitación del monumento.

\section{CONCLUSIONES}

Tras el trabajo realizado, queda de manifiesto la necesidad de combinar diferentes técnicas para poder conseguir los mejores resultados encaminados a poseer los datos que permitirán un análisis exhaustivo del edificio. Cada una de las técnicas aplicadas cubre una parcela de información particular sobre las características que se necesitan para poder evaluar los trabajos que serán necesarios en la restauración del objeto patrimonial estudiado.
En este sentido la elaboración de la cartografía de los alzados a partir de la rectificación fotográfica permite la digitalización sobre pantalla de todas aquellas grietas, roturas y zonas de degradación de los diferentes materiales utilizados en la construcción, así como el despiece de los sillares que forman los muros. En este punto se ha comprobado la gran utilidad del parámetro de la reflectividad, capturado por el láser escáner, que permite distinguir, en las tomas con poca luz, los diferentes materiales que componen los paramentos. De esta manera se puede delimitar cada uno de los materiales lo que permitió hacer el despiece de los muros interiores con mayor facilidad.

Así mismo, la utilización del color en las tomas de datos del escáner han permitido una mejor interpretación de las formas que se establecen en el espacio 3D. La técnica de aplicación de color a posteriori a las nubes de puntos y al modelo virtual tridimensional da lugar al reconocimiento de posibles patologías debidas a humedades o filtraciones que podrían llevar a un colapso de la estructura.

Para llevar a cabo estudios más complejos sobre la estructura del edificio, empleando métodos como elementos finitos, es necesario disponer de un modelo 3D. Éste ha sido generado a partir de los datos obtenidos mediante láser escáner.

Para la obtención del modelo de forma paramétrica, para su mejor análisis, ha sido determinante la utilización del cálculo de los parámetros que definen las diferentes superficies. La clasificación de la superficie y la extracción de los datos del centro y los semiejes de las superficies curvas facilitan enormemente la elaboración en las maquetas virtuales 3D.

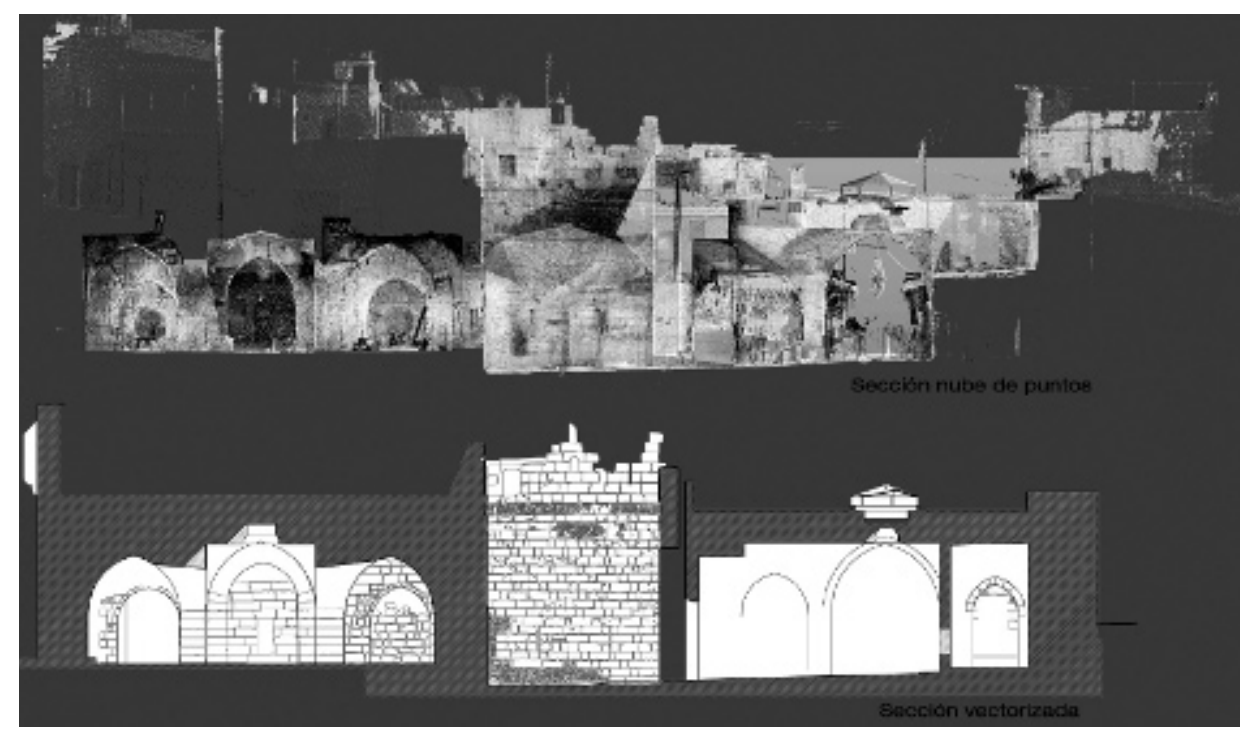


En este proceso se ha empleado el ajuste por métodos robustos. Su utilización en el filtrado de los datos ha sido determinante y ha producido mejor resultado que los métodos de mínimos cuadrados tradicionales. Esta cuestión ha permitido reducir los valores residuales y obtener precisiones más ajustadas para la clasificación de las superficies.
Una vez determinados los datos que definen las superficies y elaborada la maqueta virtual $3 \mathrm{D}$ se pueden realizar los análisis de deformaciones de elementos estructurales por desplazamientos de parte de la superficie haciendo las comparaciones entre los datos extraídos de la toma de láser escáner con los modelos teóricos tridimensionales.

\section{BIBLIOGRAFÍA}

(1) UNESCO: Convention concerning the protection of the world cultural and natural heritage. p. 16, Paris, 1972. http://whc.unesco.org/archive/convention-en.pdf.

(2) McGlone, J. Ch.: Manual of Photogrammetry. (5 $5^{a}$ ed.). ASPRS, Bethesda, Maryland, 2004.

(3) Pieris, S.; Prematilleke, P.L.: "Architectural Photogrammetry". CIPA - ICOMOS, p. 189 Colombo, Sri Lanka, 1993.

(4) Karara, H. M.: Non Topographic Photogrammetry. ASPRS, Falls Church, Virginia, 1989.

(5) Chen, Y.; Medioni, G.: "Object Modelling by Registration of Multiple Range Images". International Journal of Image and Vision Computing (IVC), Vol. 10 n³ (1992), pp. 145-155.

(6) Huising, E. J.; Gomes, L. M.: "Errors and accuracy estimates of laser data acquired by various laser scanning for topographic applications". ISPRS Journal of Photogrammetry \& Remote Sensing, Vol. 53 n5 (1998), pp. 245-261.

(7) Kilian, J.; Haala, N.; Englich, M.: "Capture and evaluation of airborne laser scanner data". Int. Arch. Photogramm. Remote Sensing XXXI, Part B3, pp: 383-388, 1996.

(8) Kraus, K.; Pfeifer, N.: "Determination of terrain models in wooded areas with airborne laser scanner data". ISPRS Journal of Photogrammetry and Remote Sensing Vol. 53 n4 (1998), pp.193-203.

(9) Rousseeuw, P. J.; Leroy, A. M.: Robust Regression and Outlier Detection. p. 329, John Wiley \& Sons, Nueva York, 1987.

(10) Fischler, M. A.; Bolles, R. C.: "Random Sample Consensus: A Paradigm for Model Fitting with Applications to Image Analysis and Automated Cartography". Comm. of the ACM, Vol. 24, (1981), pp: 381-395.

(11) Schnabel, R.; et ál.: "Efficient RANSAC for point-cloud shape detection". Computer Graphics Forum, Vol. 26 n² (2007), pp: 214-226.

(12) Busquets, J.: Aleppo: rehabilitation of the old city. Harvard University Graduate School of Design, Cambridge, 2005.

(13) Manual Riegl, http://www.riegl.com/nc/products/terrestrial-scanning/

(14) Ullrich, A.; Reichert, R.; Schwarz, R.; Riegl, J.: "Time-of-flight-based 3D imaging sensor with true-color channel for automated texturing". 5th Conference on Optical 3-D Measurement Techniques, Viena, Oct-2001.

(15) Stephan, A.; Heinz, I.; Mettenleiter, M.; Härtl, F.; Fröhlich, C.; Dalton, G.; Hines, D.: "Laser Sensor for As-Built-Documentation". Geodesy for Geotechnical and Structural Engineering II, Berlín, Mayo-2002.

(16) Akca, D.; Gruen, A.: "Least squares 3D surface and curve matching". ISPRS Journal of Photogrammetry and Remote Sensing, Vol. 59 n³ (2005), pp. 151-174.

(17) Wolf, P. R.; Ghilani, C. D.: Adjustment Computations: Statistics and Least Squares in Surveying and GIS. p. 564, Wiley Series in Surveying and Boundary Control, cidudad, 1997. 